\title{
THIN RF WINDOWS FOR HIGH-PRESSURE GAS-FILLED CAVITIES*
}

\author{
M. Alsharo'a ${ }^{\#}$, R. P. Johnson, Muons, Inc. Batavia, IL USA. \\ M. Gosz, D. M. Kaplan, S. Nair, Illinois Institute of Technology, Chicago IL USA. \\ A. Moretti, G. Romanov, Fermilab, Batavia, IL USA.
}

\begin{abstract}
RF cavities for muon ionization cooling channels can be separated by RF windows to improve the internal voltage profile in each cavity and to make the cavities independent of each other. The window material must be sufficiently transparent to muons so as not to affect the beam cooling. The windows should thus be thin and made of a low- $Z$ material. A thin, flat, beryllium window was studied in order to improve the performance of pressurized RF cavities. Electromagnetic analysis was performed to solve for the fields, frequency, quality factor, and RF power loss density. Natural convection analysis of the gas within the cavity was performed to solve for the gas temperature and the gas film coefficient. Thermal analysis of the window was performed to determine its temperature profile. For the case of pressurized RF cavities, in contrast to those that operate in vacuum, thinner and simpler window designs can be used. This is due to the external gas cooling of the window.
\end{abstract}

\section{INTRODUCTION}

Ionization cooling, in which muon energy is lost in low- $Z$ absorbers and only the longitudinal component is restored using RF cavities, is currently the best-known muon cooling technique [1]. RF cavities filled with highpressure hydrogen gas bring two advantages to the ionization cooling technique. First, the energy absorption and energy regeneration happen simultaneously rather than sequentially. Second, higher RF gradients and better cavity breakdown behavior are achieved due to the Paschen effect [2]. The linear dependence of the breakdown voltage on the pressure was verified for an $805 \mathrm{MHz}$ hydrogen-filled test cavity. A maximum stable gradient of $80 \mathrm{MV} / \mathrm{m}$, which was the surface gradient limit of the electrodes, was achieved $[2,3]$.

Closing the ends of the pressurized RF cavities by electrically conducting windows allows the cavities to operate independently and improves the internal voltage profile. The use of RF windows decreases the maximum surface electric field enhancement, which decreases field emission effects. To decrease the scattering of the beam that passes through the RF windows, the windows should be thin and made of a low- $Z$ material such as beryllium. For RF cavities for muon-cooling applications, the temperature increase in the thin windows is a major complication in the operation of the cavity [4]. This is because the temperature increase causes the windows to displace out-of-plane, which detunes the cavity. The

*Work supported in part by DOE STTR grant DE-FG02-02ER86145 \#sharo@muonsinc.com beryllium foils designed for the $805 \mathrm{MHz}$ cavity have a thickness of $127 \mu \mathrm{m}$ and a diameter of $16 \mathrm{~cm}$. It was found that the temperature increase in the foil must be kept below $27 \mathrm{~K}$ to keep the frequency shift within the tolerable limit of about $10 \mathrm{kHz}$. However, a practical solution for such flat, thin foils for cavities operating in vacuum at high gradient has not yet been found.

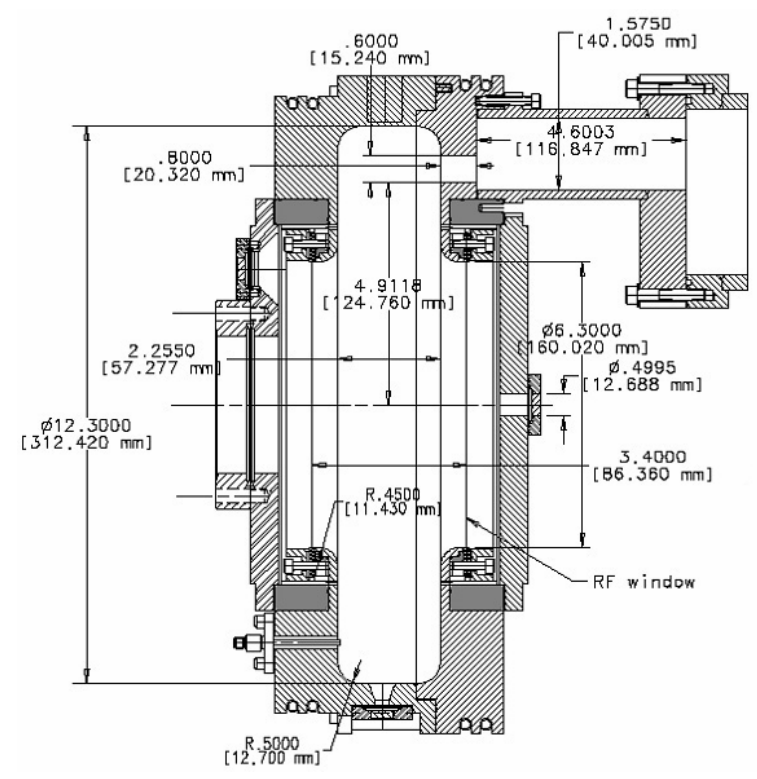

Figure 1: Geometry of the $805 \mathrm{MHz}$ cavity.

The $805 \mathrm{MHz}$ cavity shown in Figure 1 was an earlier design to test the performance of RF cavities operating in vacuum [5]. In this work, a copper cavity that has the same internal volume as the cavity shown in Figure 1 and closed by thin, flat, beryllium windows at both ends was studied. Some holes drilled in the cavity body allow the gas to pass through the cavity. It is required to keep the temperature of the gas and the windows close to $77 \mathrm{~K}$ to achieve homogeneous beam cooling. Electromagnetic and thermal analyses of the cavity and the windows were performed. The finite element analysis was carried out using ANSYS [6].

\section{ELECTROMAGNETIC ANALYSIS}

The volume of gas inside the cavity was meshed with 3-d tetrahedral elements for electromagnetic analysis. One major task was to predict the permittivity of the pressurized hydrogen gas. The frequency-pressure curve of an $805 \mathrm{MHz}$ "pillbox" cavity, pressurized with hydrogen gas, was obtained experimentally [7]. Extrapolation of the curve determined a relative 
permittivity value of about 1.12 at $77 \mathrm{~K}$ and $11 \mathrm{MPa}$. A theoretical relative permittivity value of about 1.11 was reported at the same pressure and temperature [8]. Modelling showed that the diameter of the $805 \mathrm{MHz}$ cavity, operated at vacuum, should be decreased about $4.7 \%$ to obtain a frequency of $805 \mathrm{MHz}$ when filled with hydrogen gas at $77 \mathrm{~K}$ and $11 \mathrm{MPa}$. The electric field map is shown in Figure 2. The electric field was similar to that of the cylindrical "pillbox" cavity everywhere except near the curved areas. The maximum surface electric field was approximately equal to the on-axis electric field. The quality factor of the cavity was $200 \%$ that of the cavity operating at room temperature. This was due to the decrease in RF power losses, which was due to the decrease in the resistivity of both beryllium and copper. The dimensions, properties, and electromagnetic parameters are summarized in Table 1.

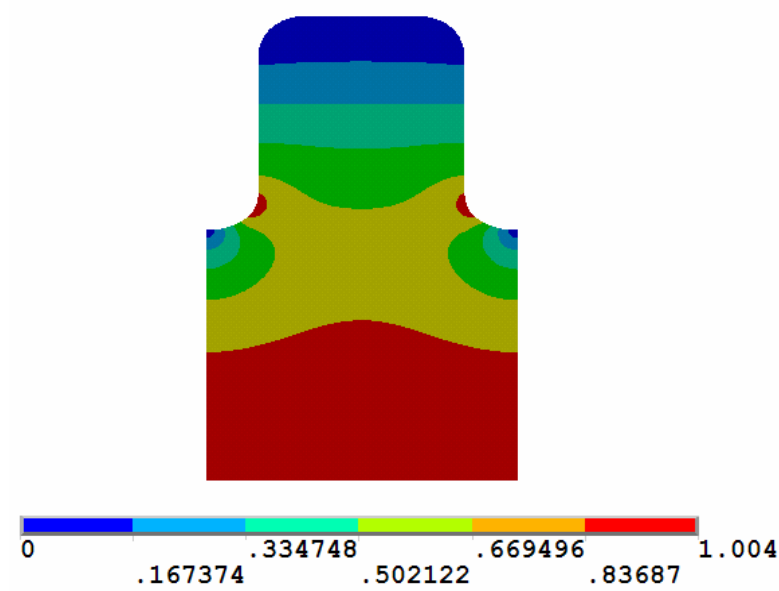

Figure 2: Electric field map (normalized).

\section{THERMAL ANALYSIS}

The thermal analysis involved three steps. The first step was a convection analysis, whereby the natural convection due to the deposition of beam energy in the gas was modelled to obtain both the gas temperature distribution and the gas film coefficient. The second step was an electromagnetic-thermal analysis, whereby the RF power loss density due to the electromagnetic radiation inside the cavity was obtained. The third step was thermal conduction analysis, whereby the temperature profile in the window was determined.

\section{Modelling of Natural Convection}

A two-dimensional model of the cavity, represented by a circle of $14.8 \mathrm{~cm}$ radius, was used to model the natural convection of the gas due to the deposition of beam energy. The beam energy was considered as internal heat generation acting throughout a circle of $8 \mathrm{~cm}$ radius and with a center that coincides with the center of the cavity. The temperature of the cavity wall was assumed to be 77 $\mathrm{K}$.
Table 1: Dimensions, properties, and parameters.

\begin{tabular}{|c|c|}
\hline Parameter & Value \\
\hline Diameter of the cavity $(D)$ & $29.7 \mathrm{~cm}$ \\
\hline Radius of the RF window $(R)$ & $8.0 \mathrm{~cm}$ \\
\hline Thickness of the RF window (t) & $127.0 \mu \mathrm{m}$ \\
\hline Gas reference pressure $(P)$ & 11.0 MPa \\
\hline Gas reference temperature $(T)$ & $77.0 \mathrm{~K}$ \\
\hline Gas density $(\rho)$ & $34.7 \mathrm{~kg} / \mathrm{m}^{3}$ \\
\hline Gas kinematic viscosity $(v)$ & $1.58 \times 10^{-7} \mathrm{~m}^{2} / \mathrm{s}$ \\
\hline Gas specific heat $\left(c_{p}\right)$ & $1.55 \times 10^{4} \mathrm{~J} / \mathrm{kg} . \mathrm{K}$ \\
\hline Gas thermal conductivity ( $K$ ) & $0.098 \mathrm{~W} / \mathrm{m} . \mathrm{K}$ \\
\hline Gas thermal diffusivity $(\alpha)$ & $1.82 \times 10^{-7} \mathrm{~m}^{2} / \mathrm{s}$ \\
\hline Gas coefficient of thermal expansion $(\beta)$ & $0.01299 K^{-1}$ \\
\hline Heat generation per unit volume $\left(q^{\prime \prime \prime}\right)$ & $3128.6 \mathrm{~W} / \mathrm{m}^{3}$ \\
\hline Prandtl number $(P r)$ & 0.87 \\
\hline Rayleigh number $(R a)$ & $2.0 \times 10^{14}$ \\
\hline Average Nusselt number $(\mathrm{Nu})$ & 218.0 \\
\hline Film coefficient $(h)$ & $79.2 \mathrm{~W} / \mathrm{m}^{2} . \mathrm{k}$ \\
\hline On-axis electric field $\left(E_{0}\right)$ & $30.0 \mathrm{MV} / \mathrm{m}$ \\
\hline Frequency $(f)$ & 805.0 MHz \\
\hline Quality factor $(Q)$ & $5.6 \times 10^{4}$ \\
\hline Electrical conductivity-beryllium & $2.56 \times 10^{9}(\Omega . m)^{-1}$ \\
\hline Electrical conductivity-copper & $4.61 \times 10^{8}(\Omega . m)^{-1}$ \\
\hline
\end{tabular}

The following are the dimensionless parameters:

$$
\begin{gathered}
\operatorname{Pr}=\frac{v}{\alpha}, \\
R a=\frac{\beta q^{\prime \prime \prime} D^{5} \rho c_{p} g}{K^{2} v}, \\
N u=\frac{h D}{K} .
\end{gathered}
$$

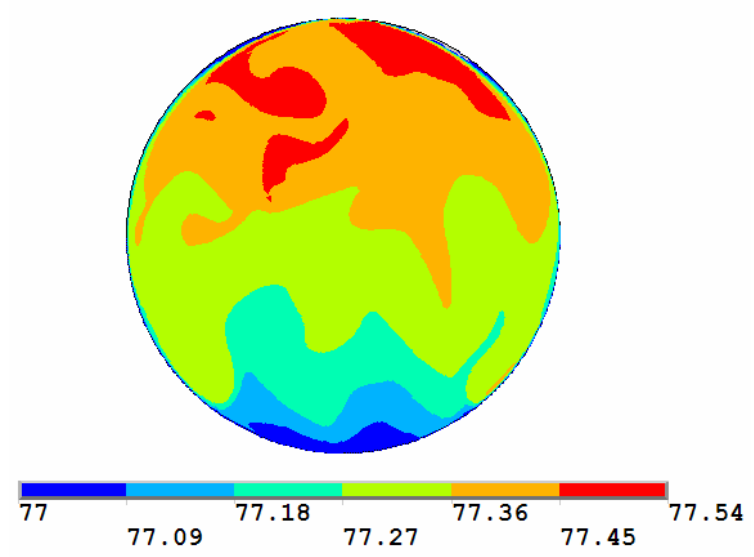

Figure 3: Contour plot of the gas temperature (K).

The thermal properties [8], non-dimensional parameters, and film coefficient are reported in Table 1. $R a$ was higher than the critical $R a$ (about $1 \times 10^{10}$ ), which indicated the occurrence of turbulent flow. Figure 3 
shows the temperature distribution in the gas. To verify the natural convection results, many cases with various values of Rayleigh number were modelled and then compared to liquid-hydrogen convection results [9] as shown in Figure 4. The difference in slope between the liquid and gaseous hydrogen data is due to the difference in thermal properties such as Prandtl number.

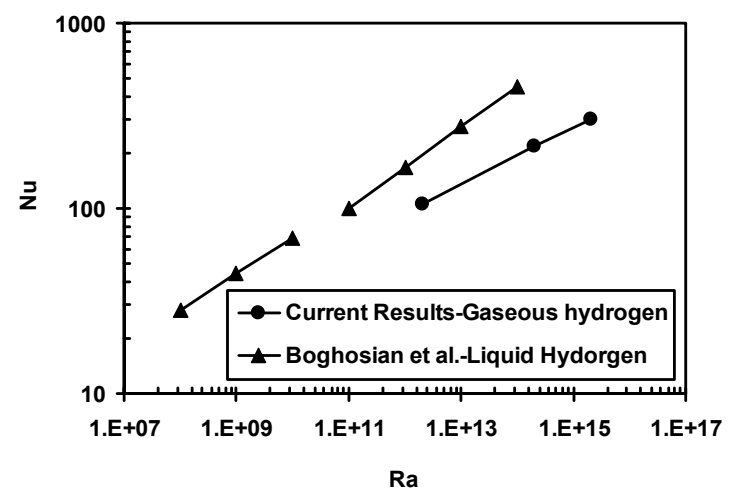

Figure 4: Dependence of the average Nusselt number on the Rayleigh number for beam heating of gaseous and liquid hydrogen in a cylinder (2-d analysis).

\section{Thermal Conduction Analysis}

The window was meshed with thermal surface elements to solve for the power loss density. The results of the electromagnetic analysis were used to calculate the RF power loss density in the window. The RF power loss density is shown in Figure 5. The window was then meshed with thermal shell elements. The convection loads and RF power loss density were applied to the model. The temperature distribution is shown in Figure 6. The negligible temperature rise in the window indicates safe thermal operation. The finite element procedure employed in this work was verified by comparing finite element results with analytical results for a "pillbox" cavity as reported in [10].

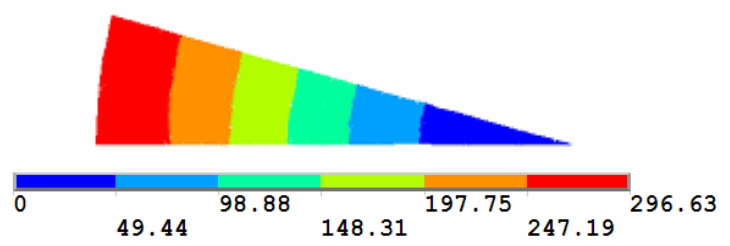

Figure 5: RF power loss density in the window $\left(\mathrm{W} / \mathrm{m}^{2}\right)$. $1 / 24^{\text {th }}$ of the full circular model.

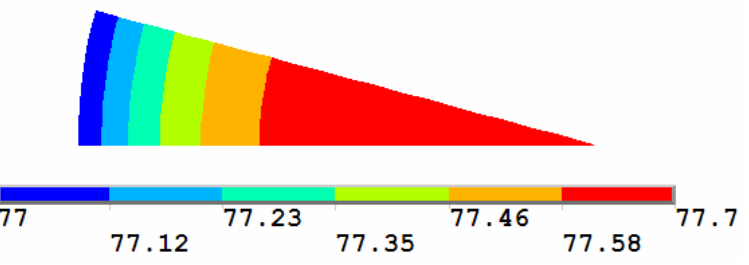

Figure 6: Temperature distribution in the window (K). $1 / 24^{\text {th }}$ of the full circular model.

\section{CONCLUSIONS}

The use of beryllium RF windows was studied for pressurized cavities from both electromagnetic and thermal points of view. An $805 \mathrm{MHz}$ cavity, filled with hydrogen gas at $77 \mathrm{~K}$ and $11 \mathrm{MPa}$, was modelled. The quality factor of the cavity was increased by a factor two compared with that of the same cavity operated at room temperature. Turbulent natural convection analysis of the gas was performed to calculate the gas temperature and the gas film coefficient. The temperature increase in the gas was negligible, indicating homogeneous beam cooling. The temperature rise in a window of $127 \mu \mathrm{m}$ thickness due to RF power losses and the deposition of beam energy in the gas was negligible (less than $1{ }^{\circ} \mathrm{C}$ ). The advantages of the present window-cavity design are that the window improves the internal voltage profile and the external gas cooling decreases the temperatures rise in the window.

\section{REFERENCES}

[1] M. Alsharo'a et al., "Recent Progress in Neutrino Factory and Muon Collider Research Within the Muon Collaboration", Phys. Rev. STAB 6 (2003) 081001.

[2] R. P. Johnson et al., "Gaseous Hydrogen and Muon Accelerators", International Workshop on Hydrogen in Materials and Vacuum Systems, Newport News, Virginia, November 2002. Published in AIP Conf. Proc. 671 (2003) 328.

[3] R. P. Johnson et al., "High Pressure, High Gradient Cavities for Muon Cooling", XXII International Linac Conference, Lübeck, Germany, August 2004.

[4] J. N. Corlett et al., "Finite Element Analysis of Thin Beryllium Windows for a Muon Cooling Channel", PAC'01, Chicago IL, June 2001, p. 909.

[5] D. Li et al., "Design and Fabrication of an $805 \mathrm{MHz}$ RF Cavity with Beryllium Windows for a High Power RF Testing for a Muon Cooling Experiment", PAC'01, Chicago IL, June, 2001. p. 918.

[6] ANSYS Finite Element Software, ANSYS Inc.

[7] R. E. Hartline et al., "Mark II High-Pressure RF Test Cell Measurements with Molybdenum Electrodes at Lab G”, Muon Collider Note Number: MUC-NOTECOOL_EXP-0285, 2004.

[8] R. D. McCarty, "Hydrogen Technological SurveyThermophysical Properties", Scientific and Technical Office, National Aeronautics and Space Administration, Washington, DC, 1975.

[9] M. Boghosian, "A Numerical Investigation of Laminar and Turbulent Natural Convection in A Horizontal Cylinder with Internal Heat Generation", M.S. Thesis, Mechanical, Materials and Aerospace Engineering Department, Illinois Institute of Technology, July 2001.

[10]M. Alsharo'a, "Electromagnetic and Mechanical Design of Gridded RF Cavity Windows", Ph.D. Thesis, Mechanical, Materials and Aerospace Engineering Department, Illinois Institute of Technology, December 2004. 